\title{
Outline of a logic of knowledge of acquaintance
}

\author{
S. Iaquinto (Università di Milano), G. Spolaore (Università di Padova) \\ Penultimate draft. Final version to appear in Analysis.
}

\begin{abstract}
The verb 'to know' can be used both in ascriptions of propositional knowledge (e.g., 'Mary knows that Smith smokes') and ascriptions of knowledge of acquaintance (e.g., 'Mary knows Smith'). In the formal epistemology literature, the former use of 'know' has attracted considerable attention, while the latter is typically (dis)regarded as derivative. This attitude may be unsatisfactory for those philosophers who, like Russell, are not willing to think of knowledge of acquaintance as a subsidiary or dependent kind of knowledge. In this paper we outline a logic of knowledge of acquaintance in which ascriptions like 'Mary knows Smith' are regarded as formally interesting in their own right, remaining neutral on their relation to ascriptions of propositional knowledge. The resulting logical framework, which is based on Hintikka's modal approach to epistemic logic, provides a fresh perspective on various issues and notions at play in the philosophical debate on acquaintance.
\end{abstract}

1. In contemporary epistemology, it is common to distinguish between three kinds of knowledge (see, e.g., Bengson and Moffett 2012, Fantl 2016, Ichikawa and Steup 2017). Speaking roughly, these are (i) cases of knowledge in which a cognitive agent $\mathrm{S}$ knows that something is true-for example, that the Earth revolves around the Sun, (ii) cases in which S knows how to do something-for example, how to ride a bicycle, and (iii) cases in which $\mathrm{S}$ knows a given thingfor example, a given person. For the present purposes, we focus on the first kind 
of knowledge — the so-called knowledge-that—and the third one—which we are going to call knowledge of acquaintance (or acquaintance for brevity) 1

We assume that statements like 'Mary knows Smith' are ascriptions of (knowledge of) acquaintance just as statements like 'Mary knows that the Earth revolves around the Sun' are ascriptions of knowledge-that. Starting from Hintikka's (1962) seminal work, over the last few decades a wide range of intriguing formalisations have been proposed for knowledge-that ascriptions (see van Benthem 2006 for an account). In comparison, ascriptions of the second kind have received much less attention. More importantly, scholars working within the formal epistemology tradition have generally followed Hintikka in regarding ascriptions of acquaintance as either reducible or otherwise dependent on ascriptions of knowledge-that $\left.\right|^{2}$ The resulting treatments have proved fruitful from both a linguistic and a logical perspective. However, they are not philosophically neutral. As Hintikka (1970: 883) himself remarks,

[my views] are incompatible with many well-known philosophical doctrines. To mention only one, if my analysis $[\ldots]$ of the directobject construction with 'knows' is essentially correct, I have in a

1 The label 'knowledge of acquaintance' is due to Grote 1865 ) and has been popularised by James (1885). We prefer it to Russell's (1910,1912) 'knowledge by acquaintance', which is generally used to denote a kind of knowledge-that.

${ }^{2}$ Hintikka proposed to analyse knowledge of acquaintance as a kind of de re knowledge-that: informally, 'S knows $o$ ' becomes 'For some $x$, S knows that $o=x$ '. See Hintikka 1970 878883 and 1991 212-233. Hintikka's formal treatment of acquaintance ascriptions is based on his previous work on knowing-who ascriptions. See, for instance, Hintikka 1962 131-132, 148-150 and 1969 49-50, 96-98. See also Böer and Lycan 1986 and Hintikka 1996 For recent work building on Hintikka's approach to acquaintance and knowing-who, see Aloni 2017 
sense disproved Russell's claim [that knowledge of acquaintance] is "logically independent of knowledge of truths." For in [my analysis] the only construction in which 'knows' occurs is (a shorthand for) 'knows that', which surely expresses "knowledge of truths."

The goal of this paper is to define a framework within which it is possible to express and formally study alternative conceptions of knowledge of acquaintance and its epistemological role, including Russell's own stance. We assume a traditional, Hintikka-style treatment of knowledge-that ascriptions, and we introduce acquaintance ascriptions as objects of formal study in their own right, making no assumption as to whether they reduce to, or depend on, ascriptions of knowledgethat. Within this general framework, we consider different constraints that may be put on acquaintance and try to set out as clearly as possible where they lead.

Our formal proposal, although simple and philosophically neutral, allows the proof of some nontrivial results about the relationship between knowledge of acquaintance and knowledge-that, and it grants a fresh take on a few notions at play in the epistemological debate on the foundations of knowledge.

2. Before introducing our proposal, let us briefly recall some notions of (propositional) epistemic modal logic.

The language (let us call it $\mathcal{L}_{K}$ ) consists of countably many propositional variables ' $p$ ', ' $q$ ', .. ; unary connectives $\neg$ and $K$; a binary connective $\wedge$ (other connectives are defined in the usual way); and parentheses. Sentences $\varphi$ of $\mathcal{L}_{\mathcal{K}}$ may be formally specified as:

$$
\varphi::=p|\neg \varphi|(\varphi \wedge \varphi) \mid K \varphi .
$$


Sentences of form $K \varphi$ are the formal counterparts of ascriptions of knowledgethat to a given agent $\mathrm{S}$. $K \varphi$ reads 'S knows that $\varphi$ ' or, taking the identity of $\mathrm{S}$ as irrelevant, 'It is known that $\varphi$ '.

Sentences of $\mathcal{L}_{K}$ are evaluated on Kripke models $\mathfrak{M}_{K}=\left(W, R_{K}, \sigma\right)$, where $W$ is a nonempty set (intuitively, the set of all possible worlds); $R_{K}$ is a binary reflexive relation on $W$, and $\sigma$ is an evaluation function that associates each propositional variable $p$ with a subset of $W$ (intuitively, the set of worlds in which $p$ is true).

The notion of a sentence $\varphi$ being true in model $\mathfrak{M}_{K}$ in world $w\left(\mathfrak{M}_{K}, w \vDash \varphi\right.$ in symbols) is recursively defined as follows:

[i] If $p$ is a propositional variable, $\mathfrak{M}_{K}, w \vDash p$ iff $w \in \sigma(p)$;

[ii] $\quad \mathfrak{M}_{K}, w \vDash \neg \varphi$ iff $\mathfrak{M}_{K}, w \not \models \varphi$;

[iii] $\mathfrak{M}_{K}, w \vDash \varphi \wedge \psi$ iff $\mathfrak{M}_{K}, w \vDash \varphi$ and $\mathfrak{M}_{K}, w \vDash \psi$;

[iv] $\mathfrak{M}_{K}, w \vDash K \varphi$ iff $\mathfrak{M}_{K}, v \vDash \varphi$ for each world $v \in W$ such that $w R_{K} v$.

Throughout the paper, we assume that logical truth and consequence are defined in the usual way.

Intuitively, $R_{K}$ is an accessibility relation that holds between worlds $w, v \in W$ if and only if $v$ is compatible with what $\mathrm{S}$ knows to be true in $w$. In turn, a world $v$ is compatible with what $\mathrm{S}$ knows in $w$ if and only if all the propositions that $\mathrm{S}$ knows to be true in $w$ are true in $v$. Therefore, by the reflexivity of $R_{K}$, all propositions that $\mathrm{S}$ knows to be true in $w$ are true in $w$. This is in order to express the idea that knowledge is factive, viz., that if $\mathrm{S}$ knows that a proposition is true then that proposition is true. 
3. Now we have the tools to bring out some analogies between knowledge-that, as dealt with in modal epistemic logic, and knowledge of acquaintance. Our guiding thought is that, intuitively, knowledge-that is to the truth of known propositions just as knowledge of acquaintance is to the existence of known entities. Existence, in our sense, is a property that is not trivially possessed by all entities in all worlds. Moreover, we ignore tense-related complexities and use 'to exist' as equivalent to 'to exist at some time'. Apart from that, what we mean by 'existence' may vary depending on the kind of entities at play in acquaintance ascriptions. For instance, if the objects of acquaintance are understood to be ordinary things from the 'external' world (see, e.g., Johnston 2004) we take 'existence' to mean concrete or spatiotemporal existence. Among the objects of acquaintance that can be said to exist (or, better, to subsist) in this sense, one may also find facts, conceived of as nonlinguistic, mind-independent objects (see Fumerton 1995: 73). In contrast, some philosophers hold that knowledge of acquaintance is (primarily) directed towards 'internal' or phenomenal entities like sense data or pains (see Russell 1910, Russell 1912; Ch. 5, Price 1950, and Robinson 1994); for our purposes, we may assume that phenomenal entities exist if, and only if, they are perceived. In presenting our formal framework, we remain philosophically neutral on the issue of what objects are (or can be) known.

Bearing this in mind, let us start by assuming the following, plausible thesis:

(A) If $\mathrm{S}$ knows an object $o$, then $o$ exists. 
Thesis (A) says that the existence of $o$ is a necessary condition for knowing $o 3^{3}$ (A) is the existential analogue of factivity: knowing $o$ requires the existence of $o$ just as knowing that $p$ requires the truth of $p$. By contraposition, you cannot know $o$ if $o$ does not exist, just as you cannot know that $p$ if $p$ is false. In a way, factivity and (A) are two distinct specifications of a more general principle that, echoing Parmenides (DK, fr. 2, line 7), we could voice as 'thou couldst not know that which is not' 4

As we said above, in modelling knowledge-that, standard epistemic logic associates with a cognitive agent $\mathrm{S}$ a set of worlds representing all the situations that are compatible with what $\mathrm{S}$ knows. These worlds are sometimes called epistemic alternatives, since they represent all the different ways in which the world could be, compatibly with S's propositional knowledge. Our idea is that a similar approach can be employed to modelling knowledge of acquaintance.

We propose to associate to an agent $\mathrm{S}$ in a given world $w$ a set of possible worlds representing all the situations that are compatible with S's knowledge of acquaintance in $w$. We call these worlds objectual alternatives to $w$. We assume that a world $v$ is an objectual alternative to $w$ if and only if every object that $\mathrm{S}$ knows in $w$ exists in $v$. Hence, similarly to what we have just seen in formalising cases of knowledge-that, we shall say that $\mathrm{S}$ knows an object $o$ in a given world $w$ if and only if $o$ exists in all the objectual alternatives to $w$.

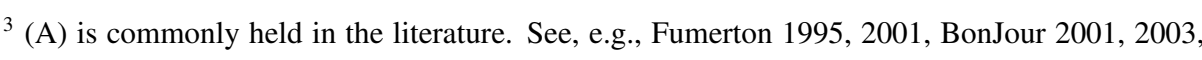
and Huemer 2007

${ }^{4}$ In Raven's translation (Kirk and Raven 1957 269). We do not presuppose we are using 'which is not' in the same sense as Parmenides.
} 
Formally, the possible objects of acquaintance are represented by a countable set $O$ whose elements $o, \pi, \ldots$ are called objectual variables. The set $P$ of propositional variables includes, for each objectual variable $o$, a corresponding existential variable $\varepsilon_{o}$. Intuitively, an existential variable $\varepsilon_{o}$ is a sentence that attributes existence to the corresponding object $o$. In other words, $\varepsilon_{o}$ reads ' $o$ exists'. The language $\mathcal{L}_{A}$ consists of $O$ and $P$, of an operator $K_{A}$, and, as before, of connectives $\neg, \wedge$, and parentheses. Sentences $\varphi$ of $\mathcal{L}_{A}$ may be formally specified as:

$$
\varphi::=p|\neg \varphi|(\varphi \wedge \varphi) \mid K_{A} O
$$

Note that, while $K$ could be put in front of every formula obtaining a new formula, here $K_{A}$ can only be put in front of an objectual variable. This is because $K_{A}$ is used to represent cases of knowledge of objects and it is not clear what the negation of an object or the conjunction of two objects is.

Sentences of $\mathcal{L}_{A}$ are evaluated on reflexive Kripke models $\mathfrak{M}_{A}=\left(W, R_{A}, \tau\right)$, where $W$ is as defined above; relation $R_{A}$ holds between worlds $w, v$ if and only if $v$ is compatible with the objectual knowledge of $\mathrm{S}$ in $w$; and $\tau$ is a function which associates every objectual or propositional variable to a subset of $W$. Intuitively, if $\gamma$ is a propositional variable, then $\tau(\gamma)$ is the set of worlds in which $\gamma$ is true; if $\gamma$ is an objectual variable, then $\tau(\gamma)$ is the set of worlds in which $\gamma$ exists. (From now on we shall use $o$ and $\varepsilon_{o}$ as (autonymous) metavariables for objectual and existential variables, respectively.) For any two variables $o$ and $\varepsilon_{o}$, we impose that $\tau(o)=\tau\left(\varepsilon_{o}\right)$, for obviously the worlds in which an object exists are the same as the ones in which the proposition that that object exists is true.$^{5}$

\footnotetext{
${ }^{5}$ It might also be natural to require that objects of knowledge of acquaintance are only contingently known, so that no object $o$ exists in all worlds in $W$. We skip this condition, however, for
} 
The notion of truth in $\mathfrak{M}_{A}, w$ is defined as follows:

[i']-[iii'] Essentially identical to clauses [i] [iii] above,

[iv'] $\mathfrak{M}_{A}, w \models K_{A} O$ iff $v \in \tau(o)$ for each $v \in W$ such that $w R_{A} v$.

Clause [iv'] says that $\mathrm{S}$ knows an object $o$ in $w$ if and only if, for each world $v$ compatible with $w, v$ is in $\tau(o)$, that is, $o$ exists in $v$. The reflexivity of $R_{A}$ ensures that all objects known in a world exist in that world (viz., the existential analogue of factivity) just as the reflexivity of $R_{K}$ within models $\mathfrak{M}_{K}$ ensures that all propositions known to be true in a world are true in that world (viz., factivity). Hence, the object-language counterpart of (A),

$\left(\mathrm{A}^{*}\right)\left(K_{A} o \rightarrow \varepsilon_{o}\right)$

is valid within our framework and for essentially the same reason that $(K \varphi \rightarrow \varphi)$ is valid in epistemic modal logic.

4. From a philosophical viewpoint, clause (A) can be refined by exploring how knowledge of acquaintance relates to knowledge-that.

Some philosophers have attributed a foundational role to knowledge of acquaintance, by taking it as a more basic and direct form of knowledge than propositional knowledge. In particular, according to Russell (1912; 25), knowledge of acquaintance

is essentially simpler than any knowledge of truths, and logically independent of knowledge of truths, though it would be rash to assume that human beings ever, in fact, have acquaintance with things without at the same time knowing some truth about them.

contingency is not expressible in $\mathcal{L}_{A}$. 
As mentioned above, our aim is to offer a proposal that is compatible with different stances on the relationships between knowledge of acquaintance and knowledge-that, including Russell's own view. More specifically, the proposal is meant to be consistent with the idea, recently defended in the literature, that knowledge of acquaintance is independent of knowledge-that, in the sense that it need not presuppose or require any specific piece of propositional knowledge (see, e.g., Fumerton 1995: 74, Fumerton[2001, BonJour|2001, 2003, and Huemer|2007).

However, we take seriously Russell's suggestion that, as a matter of fact, knowledge of an object $o$ always comes with knowledge of some truth about that object. Moreover, we think it is safe to assume that some such truths attribute to $o$ an existence-entailing property (viz., a property only existing things can have). And if S knows that $o$ possesses an existence-entailing property, only a modicum of epistemic idealisation is needed to conclude that $\mathrm{S}$ knows that $o$ exists. This conclusion squares nicely with a standard philosophical view about acquaintance, namely, that we cannot reasonably doubt the existence of what we are acquainted with (see, e.g., Russell 1912: 26). Accordingly, at the price of imposing a minimal requirement of rationality on cognising agents, we can replace (A) with the following, stronger conditional:

If $\mathrm{S}$ knows an object $o$, then $o$ exists and $\mathrm{S}$ knows that $o$ exists.

By the factivity of knowledge, this clause reduces to:

(B) If $\mathrm{S}$ knows $o$, then $\mathrm{S}$ knows that $o$ exists.

Now consider the following thesis:

(C) If $v$ is an epistemic alternative to $w$, then $v$ is an objectual alternative to $w$. 
Principle (C) says that the worlds compatible with the propositional knowledge of an agent are also compatible with the agent's knowledge of acquaintance. If we think of the amount of information of an agent as inversely proportional to the amount of worlds that are compatible with what the agent knows, (C) is equivalent to the claim that knowledge of acquaintance comes with an amount of information at most equal to that encoded by knowledge-that.

Intuitively, (B) and (C) express different conditions. It is thus very interesting to observe that, within our framework, the following proposition holds:

(B) is equivalent to (C).

The proof is easy. Left to right. Let us assume that $v$ is an epistemic alternative to $w$, that is, that all the propositions that $\mathrm{S}$ knows to be true in $w$ are true in $v$ as well. Then, by (B), for any object $o$ that $\mathrm{S}$ knows in $w$, the proposition that $o$ exists is true in $v$. Hence, all the objects that $\mathrm{S}$ knows in $w$ exist in $v$, which is sufficient to conclude that $v$ is an objectual alternative to $w$. Right to left. Let us assume that S knows object $o$ in $w$. Then, $o$ exists in all objectual alternatives to $w$. But, by (C), all epistemic alternatives to $w$ are objectual alternatives to $w$. Therefore, the proposition that $o$ exists is true in all epistemic alternatives to $w$, which is sufficient to conclude that $\mathrm{S}$ knows that $o$ exists in $w$.

In order to express (B) we need a language that includes both knowledge operators $K, K_{A}$ and both objectual and propositional (including existential) variables. Let $\mathcal{L}_{K A}$ be such a language (whose definition is easily obtained from the above definitions of $\mathcal{L}_{K}$ and $\mathcal{L}_{A}$ ). Formulae of $\mathcal{L}_{K A}$ are evaluated within models $\mathfrak{M}_{K A}=\left(W, R_{K}, R_{A}, \tau\right)$, where $W, R_{K}, R_{A}$, and $\tau$ are as defined above, with a pro- 
viso: in accordance with clause $(\mathrm{C})$, we impose that, for any $w, v$, if $w R_{K} v$ then $w R_{A} v$ (viz., $R_{K} \subseteq R_{A}$ ). The semantics of $\mathcal{L}_{K A}$ is defined in the obvious way, based on the above clauses [i] [iv], [iv'], Little reflection is needed to see that the following object-language counterpart of clause (B) is a valid schema of $\mathcal{L}_{K A}$ :

$\left(\mathrm{B}^{*}\right)\left(K_{A} o \rightarrow K \varepsilon_{o}\right)$

It is easy to see that, if the converse of $\left(\mathrm{B}^{*}\right)$,

$$
\left(K \varepsilon_{o} \rightarrow K_{A} o\right)
$$

is added as a further axiom, then the distinction between objectual and epistemic alternatives collapses. As a result, ascriptions of knowledge-that and of acquaintance are interpreted on the background of the same set of epistemic alternatives, as in Hintikka's (1970, 1991) treatment of acquaintance ascriptions (see also above, \$1]. From a philosophical viewpoint, the conjunction of $\left(\mathrm{B}^{*}\right)$ and its converse corresponds to the view that there is nothing epistemologically special about acquaintance compared with ordinary propositional knowledge.

It is interesting to observe that, in a standard modal calculus that includes $\left(\mathrm{A}^{*}\right)$ as a theorem, $\left(\mathrm{B}^{*}\right)$ straightforwardly follows from a principle that may be called transparency of knowledge of acquaintance:

(D) $\left(K_{A} o \rightarrow K K_{A} o\right)$ ('If $\mathrm{S}$ knows an object $o$, then $\mathrm{S}$ knows that $\mathrm{S}$ knows $o$ ').

The converse entailment, however, does not hold in our framework, as $\left(\mathrm{B}^{*}\right)$ is strictly weaker than $(\mathrm{D})^{6}$

\footnotetext{
${ }^{6}$ To see this, it suffices to consider the countermodel $\mathfrak{M}_{K A}=\left(W, R_{K}, R_{A}, \tau\right)$ such that $W=$ $\left\{w, v, v^{\prime}\right\}, R_{K}=\left\{(w, w),(v, v),\left(v^{\prime}, v^{\prime}\right),(w, v)\right\}, R_{A}=R_{K} \cup\left\{\left(v, v^{\prime}\right)\right\}$, and $\tau(o)=\{w, v\}$.
} 
5. Intuitively, the notion of knowledge of acquaintance involves two key elements. On the one hand, it is objectual rather than propositional; on the other, it is thought to be foundational in the sense of being independent of any prior knowledge-that. Now, within our framework, it is possible to separate the first element from the second, by isolating an epistemic notion that is propositional rather than objectual but is still foundational in an important sense, in that it depends just on (follows entirely from) knowledge of acquaintance and does not depend on any prior knowledgethat. We shall call it foundational knowledge (the other possible label, knowledge by acquaintance, is very similar to knowledge of acquaintance and might invite confusion) $]^{7}$

To express foundational knowledge, we add a unary connective $K_{F}$ to our language $\mathcal{L}_{K A}$, thus obtaining language $\mathcal{L}_{K A F} . K_{F}$ has the same syntactic behaviour as $K$ but differs from it semantically in having $R_{A}$ instead of $R_{K}$ as its underlying accessibility relation. Thus, $K_{F} \varphi$ ('S has foundational knowledge that $\varphi$ ') is true in $w$ if and only if $\varphi$ is true in all objectual alternatives to $w$. In more formal terms, the semantics of $\mathcal{L}_{K A F}$ is obtained from that of $\mathcal{L}_{K A}$ by adding the following clause:

[v] $\mathfrak{M}_{K A}, w \vDash K_{F} \varphi$ iff $\mathfrak{M}_{K A}, v \vDash \varphi$ for each $v \in W$ such that $w R_{A} v$.

Clearly, this semantics validates the following schema:

$$
\text { (E) } \quad\left(K_{F} \varepsilon_{o} \leftrightarrow K_{A} o\right)
$$

\footnotetext{
${ }^{7}$ Sometimes philosophers speak of 'foundational knowledge' to indicate a kind of non-inferential knowledge, viz., knowledge that is not obtained by any kind of inferential process. We definitely do not mean 'foundational' in this sense. To be more explicit, we regard as foundational also pieces of knowledge-that that are obtained from objectual knowledge via $(\mathrm{B})$ or other inferential principles.
} 
Principle (E) says that $\mathrm{S}$ has foundational knowledge that $o$ exists if and only if S knows $o$. (E) can be thought of as a partial specification of what foundational knowledge is.

It is very natural to require that foundational knowledge be a kind of propositional knowledge, that is, that $\left(K_{F} \varphi \rightarrow K \varphi\right)$ be valid—otherwise it would be better to give up the idea that foundational knowledge exists at all. It is worth observing that this natural requirement corresponds to condition $(\mathrm{C})$ and so is equivalent to principle (B)

The limited linguistic resources of $\mathcal{L}_{K A F}$ are sufficient to express a few philosophically interesting principles. Here are two examples:

(F) $\quad\left(K \varphi \rightarrow K_{F} \varphi\right)$

(G) $\left(K \varepsilon_{o} \rightarrow K_{F} \varepsilon_{o}\right)$ (or, equivalently, $\left(K \varepsilon_{o} \rightarrow K_{A} o\right)$ )

Schema (F) corresponds to a conception that may be labelled foundationalism: all of our propositional knowledge is ultimately based on acquaintance, in the sense that knowledge of acquaintance completely determines knowledge-that (see, e.g., Russell 1912: 26). The weaker schema $(\mathrm{G})$, which is obtained from $(\mathrm{F})$ by substitution, corresponds to a view that may be called existential foundationalism: all (singular) existential propositions are known through acquaintance, so that testimony, abduction, etc. are not, as such, legitimate sources of (singular) existential knowledge.

It is obviously possible to extend the framework outlined thus far, obtaining the resources to express other key principles in the epistemology of acquaintance. If a full predicative language with identity is adopted, for instance, it becomes possible 
to formally study intriguing principles such as:

If $\mathrm{S}$ knows objects $o$ and $o^{\prime}$, and if $o$ is the same as $o^{\prime}$, then $\mathrm{S}$ knows that $o$ is the same as $o^{\prime}$, viz., $\left(\left(K_{A} o \wedge K_{A} o^{\prime} \wedge o=o^{\prime}\right) \rightarrow K o=o^{\prime}\right)$;

If $\mathrm{S}$ knows object $o$, then $\mathrm{S}$ has de re knowledge that something is identical with $o$, viz., $\left(K_{A} o \rightarrow \exists x K o=x\right)$ (cf. Hintikka|1970; 878).

We leave this and other possible extensions of the framework for future work.

\section{References}

Aloni, M. 2017. Knowing-who in quantified epistemic logic. In Jaakko Hintikka on knowledge and game theoretical semantics, eds. H. van Ditmarsch and G. Sandu. Dordrecht: Springer (forthcoming).

Bengson, J. and M. A. Moffett. 2012. Knowing How: Essays on Knowledge, Mind, and Action. Oxford: Oxford University Press.

Böer, S. and Lycan, W. (1986). Knowing Who. Cambridge, MA: MIT Press.

BonJour, L. 2001. Toward a defense of empirical foundationalism. In Resurrecting Old-Fashioned Foundationalism, ed. M. de Paul. Lanham, MD: Rowman and Littlefield.

BonJour, L. 2003. A version of internalist foundationalism. In Epistemic Justification: Internalism vs. Externalism, Foundations vs. Virtues, eds. L. BonJour and E. Sosa. Malden, MA: Blackwell. 
Fantl, J. 2016. Knowledge how. In The Stanford Encyclopedia of Philosophy (Spring 2016 ed.), ed. E. N. Zalta. Metaphysics Research Lab, Stanford University. https://plato.stanford.edu/archives/spr2016/entries/ knowledge-how/

Fumerton, R. 1995. Metaepistemology and Skepticism. Lanham, MD: Rowman and Littlefield.

Fumerton, R. 2001. Classical foundationalism. In Resurrecting Old-Fashioned Foundationalism, ed. M. de Paul. Lanham, MD: Rowman and Littlefield.

Grote, J. 1865. Exploratio Philosophica, Part I. Cambridge: Deighton, Bell and Co.

Hintikka, J. 1962. Knowledge and Belief. Ithaca, NY: Cornell University Press.

Hintikka, J. 1969. Models for Modalities. Dordrecht: Reidel

Hintikka, J. 1970. Objects of knowledge and belief: Acquaintances and public figures. The Journal of Philosophy 67(21): 869-883.

Hintikka, J. 1991. Knowledge and the Known. Dordrecht: Springer.

Hintikka, J. 1996. Knowledge acknowledged: Knowledge of propositions vs. knowledge of objects. Philosophy and Phenomenological Research 56(2): 251275.

Huemer, M. 2007. Compassionate phenomenal conservatism. Philosophy and Phenomenological Research 74(1): 30-55. 
Ichikawa, J. J. and M. Steup. 2017. The analysis of knowledge. In The Stanford Encyclopedia of Philosophy (Spring 2017 ed.), ed. E. N. Zalta. Metaphysics Research Lab, Stanford University. https://plato.stanford.edu/archives/ spr2017/entries/knowledge-analysis/

James, W. 1885. On the function of cognition. Mind 10, old series (37): 27.

Johnston, M. 2004. The obscure object of hallucination. Philosophical Studies 120(1-3): 113-183.

Kirk, G. S. and J.E. Raven. 1957. The Presocratic Philosophers. Cambridge: Cambridge University Press.

Price, H. H. 1950. Perception (2nd ed.). London: Methuen.

Robinson, H. 1994. Perception. London: Routledge.

Russell, B. 1910. Knowledge by acquaintance and knowledge by description. Proceedings of the Aristotelian Society 11: 108-128.

Russell, B. 1912. The Problems of Philosophy. London: Williams \& Norgate. Republished as The Problems of Philosophy, Oxford University Press, Oxford 1998.

van Benthem, J. 2006. Epistemic logic and epistemology: The state of their affairs. Philosophical Studies 128(1): 49-76. 